\title{
COLOR MATCHING OF A SINGLE SHADE STRUCTURALLY COLORED UNIVERSAL RESIN COMPOSITE WITH THE SURROUNDING HARD DENTAL TISSUES
}

\author{
Mona I. Riada ${ }^{*}$, Wael M. Gamal ${ }^{* *}$ and Ahmad S. Morsy ${ }^{* * *}$
}

\begin{abstract}
Color matching between the resin composite and the teeth is the most aimed target by the patient to evaluate the quality of the treatment.

The aim of this study was to investigate the ability of the uni-shade restorative material to match the tooth shade and the blending effect of the single shade structurally colored universal resin composite.

Materials and methods disc shaped specimen $(6 \times 2 \mathrm{~mm})$ were prepared from Omnichroma ${ }^{\circledR}$ single shade resin composite. Color shade was recorded for 10 freshly extracted premolars by Easyshade $^{\circledast}$ according to CIE Lab system. Standardized class V cavities $(4 \times 2 \times 1.5 \mathrm{~mm})$ were prepared on the buccal surface of each tooth away $0.5 \mathrm{~mm}$ from the CEJ. Cavities were then restored with resin composite, cured for 40s, and their color was recorded after 24 hours storage in distal water. Two tests have been done; the first was to determine the differences of the color parameters between disc and resin composite in teeth (T1). The second was to record the change in color between the sound teeth and the resin composite in teeth (T2). The color change data statistically analyzed using paired t-test. It was considered statistically significant at $(\mathrm{P}=0.05)$.
\end{abstract}

Results All color parameters in the two tests showed significant changes except $(\Delta \mathrm{L})$ in $\mathrm{T} 2$ which showed nonsignificant differences. The specimens showed decrease in lightness and showed shifting toward the green and yellow direction, while in T2 they became lighter than the teeth and directed toward the green and blue scale.

Conclusion OMNICHROMA single shade resin composite matches the shade of enamel.

KEYWORDS: Blending effect, Color match, Color parameters, Single shade resin composite.

\footnotetext{
* Professor of Restorative Dentistry, Faculty of Dentistry, Cairo University.

** Lecturer of Operative Dentistry, Faculty of Dentistry, Modern University for Technology and Information (MTI).

*** Lecturer of Operative Dentistry, Faculty of Dentistry, Al-Azhar University.
} 


\section{INTRODUCTION}

Searching for an ideal esthetic material for restoring teeth has resulted in significant improvements in esthetic materials and techniques ${ }^{1}$. Resin composite had been proved to be the excellent esthetic and tooth-colored restorative materials of choice for many dentists due to their high acceptance by patients and their excellent esthetic properties ${ }^{2}$.

The color matching between resin composite and the dental structure is the most aimed target by the patients to evaluate the quality of the aesthetic treatments, influencing decisively the judgment of the professional competence and the degree of satisfaction of the patient at the end of the treatment ${ }^{3}$.

Blending effect (BE) of esthetic dental materials refers to interrelation of these materials or materials and hard dental tissues, manifested by smaller color difference if they were observed together than if viewed in isolation ${ }^{4}$.

The blending-effect of shaded resin-composites related to restoration size as the $\mathrm{BE}$ of the fillings increased with the decrease of restoration size and the increase of the filling material translucency. The BE also increased when the color-difference between the restoration and the surrounding walls decreased ${ }^{5}$.

The color and appearance of a tooth is a complex phenomenon, with many factors such as lighting conditions, translucency, opacity, light scattering, gloss, and the human eye and brain influencing the overall perception of tooth color ${ }^{6}$. Enamel and dentin have different structural characteristics and, consequently, they exhibit different light wave characteristics. Due to its highly mineralized prismatic structure, low organic content and a small amount of water, enamel has a higher transmission of light than dentin; dentin has less mineral content, an organic tubular structure, higher water content and is less translucent ${ }^{7}$. The color of tooth and dental restorations is determined by the effect of underlying substances, which indicates that the apparent color is the result of diffuse reflectance from the inner dentine or opaque material layer through the outer translucent layer. In addition to three primary color attributes such as value, hue, and chroma, many other optical properties of tooth, such as translucency, opacity, opalescence, surface gloss and fluorescence, should be considered ${ }^{2}$.

Extensive efforts were made over the years to develop the esthetic properties of dental restorative materials. To obtain the ideal esthetics, restorative material must simulate the natural tooth in color, translucency, and surface texture, and it must show long time color stability ${ }^{8}$.

Although there has been an excellent evolution in recent years regarding composition, color availability, transparency levels and effects, these improvements have in some way improved the physical, mechanical and optical properties of the resin composite, but due to the inherent material, the resin composite still have lower biomimetic potential because they do not constitute a crystalline structure and because of the reduction of translucency, opacity, effects and absence or presence of fluorescence of many restorative systems. These characteristics alone make the color matching between the resin composite and the tooth structure much difficult to be obtained ${ }^{9}$.

Dental materials that exhibit color shifting toward the color of the surrounding hard dental tissues may have a clinical advantage as this can improve the esthetic appearance of the restoration, simplify shade matching, reduce the number of shade guide tabs, and compensate for color mismatches to some extent $^{10}$.

OMNICHROMA is a single shade structurally colored universal composite designed for use with most direct restorative clinical cases. Its wide colormatching ability eliminates the need for a shadetaking procedure and reduces composite inventory, allowing clinicians to minimize chair time, the wastage of unused composite shades, and reduce reliance on shade-matching procedures ${ }^{9}$. The null hypothesis was to investigate if single shade structurally colored universal resin composite will modify its shade to match the tooth structure shade. 


\section{MATERIALS AND METHODS}

\section{Materials used in the study}

The type, composition and manufacturer of the used restorative material are shown in (Table 1).

\begin{tabular}{|c|c|c|c|c|}
\hline Material & Specifications & Composition & Manufacturer & Batch number \\
\hline Omnichroma & $\begin{array}{l}\text { light-cured, } \\
\text { radiopaque single } \\
\text { shade universal } \\
\text { composite }\end{array}$ & $\begin{array}{l}\text { The Filler System: } \\
79 \% \text { by weight ( } 68 \% \text { by volume) of spherical silica- } \\
\text { zirconia filler (mean particle size: } 0.3 \mu \mathrm{m} \text {, particle size } \\
\text { range: } 0.2 \text { to } 0.6 \mu \mathrm{m} \text { ) and composite filler. } \\
\text { The Resin System } \\
\text { (UDMA), (TEGDMA). } \\
\text {-Mequinol, Dibutyl hydroxyl toluene and UV absorber. }\end{array}$ & $\begin{array}{c}\text { Tokuyama } \\
\text { Dental, } \\
\text { Tokyo, } \\
\text { Japan }\end{array}$ & 009 E39 \\
\hline
\end{tabular}

\section{Primary color of Omnichroma:}

Disc-shaped specimen was prepared using a custom-made plexiglass mold of internal diameter of $6 \mathrm{~mm}$, and height $2 \mathrm{~mm}$. Resin composite was compacted into it using a Teflon coated plastic filling instrument and the excess was carefully removed with an explorer. A celluloid strip was sandwiched between the upper surface of the compacted material and a glass slide of $1 \mathrm{~mm}$ thickness placed to flatten the surface, extrude excess material and to produce a smooth surface. Following compaction of the resin, the sample was then cured for 40 seconds through the mylar strip and glass slide using LED (3M EliparDeepCure-S LED USA; light intensity of $400 \mathrm{~mW} / \mathrm{cm}^{2}$, in a uniform continuous curing mode). The light intensity was checked every five samples.

The primary color of Omnichroma then was recorded using the digital spectrophotometer (Vita Easyshade $^{\circledR \mathrm{V}}$, Compact, Vita, Zahnfabrik, Bad Sackingen, Germany). Color measurements were performed by positioning the specimen on a white background to prevent potential absorption effects. The probe tip was then placed perpendicular at the center of specimen and flushed into the surface of the specimen.
To identify if the primary color of Omnichroma changed to simulate the shade of teeth when used for teeth restorations, freshly extracted 20 human premolars with different shades were collected for the study protocol. These teeth were extracted due to orthodontic demands or periodontal treatment plane. All teeth were cleaned from any calculus deposits or soft tissue debris using an ultrasonic scaler, polished with a prophylaxis paste using a polishing brush. They were microscopically examined at 20x magnification to assure the absence of cracks or defects. Teeth with cracks, caries, or both were excluded from the study. The color values of the gingival one third of buccal surface of teeth were recorded using a digital spectrophotometer. The probe tip was then placed perpendicular at the center of the gingival third and flushed onto the buccal surface of the tooth to obtain accurate measurements.

Standardized outline of a trapezoidal Class V cavity preparation of dimensions $4 \mathrm{~mm}$ width, $2 \mathrm{~mm}$ length and $1.5 \mathrm{~mm}$ depth were prepared on the buccal surface of each tooth was measured using periodontal probe, $0.5 \mathrm{~mm}$ coronal to cementenamel junction using No.56 fissure carbide bur (Komet, Germany), in a high speed hand piece with air water spray. The bur was replaced by a new one 
after every 4 cavities. The cavities were prepared by a single operator. A45-degree bevel was placed on the occlusal wall of the cavity.

All the prepared cavities were rinsed thoroughly with water, and air-dried. The whole cavity of each tooth was acid etched with $37 \%$ phosphoric acid gel (SDI)for 20 seconds, then rinsed with water spray and dried gently with air stream. The bonding agent (Bond Force, Tokuyama Dental, Tokyo, Japan)was applied according to the manufacturer's instructions to the etched cavity with a brush then air-thinned gently with air stream and light-cured for 20 seconds. Restorative material were packed in the prepared cavities using a Teflon coated plastic filling instrument and the restoration was covered with celluloid strip to minimize the oxygen inhibition layer and to obtain the smoothest possible surface, then light-cured for 40 seconds. The excess material was excised with scalpel. The specimens were then stored for 24 hours in distal water.

The specimens were gently air dried and the color values of the restorative material were recorded using spectrophotometer.

The color changes $(\Delta E)$ were calculated from the changes in CIE L, a, and b values $(\Delta L, \Delta a, \Delta b)$ as follows:

$$
\Delta E=\left[(\Delta L)^{2}+(\Delta a)^{2}+(\Delta b)^{2}\right]^{1 / 2}
$$

CIE Lab is expressed by the $\mathrm{L}$ coordinate representing color luminosity, varying from white to black, $\mathrm{a}$ and $\mathrm{b}$ coordinates representing the chromaticity of the color, with axes varying from green to red and blue to yellow, respectively.

The mean of the values obtained were calculated, and the $\mathrm{L}, \mathrm{a}$, and $\mathrm{b}$ parameters were determined.

The color change data were statistically analyzed using paired t-tests. For all statistical tests, a result was considered statistically significant at $\mathrm{P}=0.05$. All statistical calculations were carried out by Microsoft Excel (version 7).

\section{RESULTS}

Table 2: Comparison between Omnichroma primary color and as a tooth restoration

$\mathrm{L}, \mathrm{a}, \mathrm{b}$, means, and standard deviations were shown in Table (2).

TABLE (2): Mean and Standard deviation of color parameters and mean difference between the disc and restorations

\begin{tabular}{|l|c|c|c|}
\hline & $\mathrm{L}$ & $\mathrm{a}$ & $\mathrm{B}$ \\
\hline Disc & 100 & 6.5 & 403 \\
\hline Restoration & $87.17 \pm 2.09$ & $0.86 \pm 049$ & $22.21 \pm 2.75$ \\
\hline$\Delta$ & $-12.83 \pm 2.8$ & $-5.64 \pm 0.8$ & $17.91 \pm 3.1$ \\
\hline P value & $1.23 \times 10^{-8^{*}}$ & $5.02 \times 10^{-11^{*}}$ & $7.03 \times 10^{-9 *}$ \\
\hline
\end{tabular}

$\Delta L$ values: -

There was a highly significant change in the brightness $(\Delta \mathrm{L})(\mathrm{p}<0.001)$. A positive $\Delta \mathrm{L}$ indicates that the specimens became lighter, whereas a negative $\Delta \mathrm{L}$ indicates that the specimens became darker. $\Delta \mathrm{L}$ mean $-12.83 \pm 2.8$. All the specimens are negative, and the resin material became darker.

\section{$\triangle a$ values:}

There was highly significant change in $\Delta \mathrm{a}$ (p $<0.001)$. A negative $\Delta \mathrm{a}$ indicates a shift towards green color, whereas a positive $\Delta \mathrm{a}$ indicates a shift towards red color. $\Delta$ a mean $-5.64 \pm 0.8$. All the specimens showed negative $\Delta \mathrm{a}$ which indicates that shade is directed toward green.

\section{$\Delta$ b values:}

A significant change was noted for all the specimens $(\mathrm{p}<0.001)$. A positive $\Delta \mathrm{b}$ indicates a shift towards yellow color, while a negative $\Delta \mathrm{b}$ denotes a shift towards blue color. $\Delta b$ was in the range (21 - 15.6) mean 17.91 \pm 3.1. All the specimens tested showed positive $\Delta b$, which indicates that shade is directed toward yellow. 
Color changes $(\Delta \mathrm{E})$ of the resin material were ranged from $18.98-24.94$ and mean $(22.9 \pm 4.1)$.

Differences in color between tooth and restoration were shown in Table (3).

TABLE (3): Mean and Standard deviation of color parameters and mean difference between the teeth and restoration.

\begin{tabular}{|l|c|c|c|}
\hline & $\mathrm{L}$ & $\mathrm{A}$ & $\mathrm{B}$ \\
\hline Tooth & $82.36 \pm 4.82$ & $1.83 \pm 0.7$ & $30.05 \pm 2.32$ \\
\hline Restoration & $87.17 \pm 2.09$ & $0.86 \pm 049$ & $22.21 \pm 2.75$ \\
\hline$\Delta$ & $4.81 \pm 4.76$ & $-0.97 \pm 0.55$ & $-7.84 \pm 3.7$ \\
\hline P value & 0.010955 & $0.000557^{*}$ & $0.000108^{*}$ \\
\hline
\end{tabular}

\section{$\Delta L$ values}

Statistically, the study of the compared specimens showed a close match of the restoration with the tooth. The degree of matching between the tooth and the restoration is $95.4 \%$. The restoration is lighter than tooth. There was a non-significant change in the brightness $\left(\mathrm{L}^{*}\right)(\mathrm{p}<0.001) . \Delta \mathrm{L}$ was in the range $(0.4-15.5)$ mean $4.81 \pm 4.76$. All the specimens are positive, so the resin material appears lighter than teeth.

\section{$\Delta a$ values}

The study of the compared specimens showed mismatch in color of the restoration to the tooth in the $a^{*}$ values of the restoration. The degree of matching between the restoration and the tooth is $45.5 \%$. There was a significant change in $a^{*}(p<$ $0.001) . \Delta \mathrm{a}$ was in the range (-.6-0.3) mean $-0.97 \pm$ 0.55 . All the specimens showed negative $\Delta$ a which indicates that the restoration is shifted toward green.

\section{$\Delta b$ values}

The study of the compared specimens showed matching in color of the restoration and the tooth in the $b$ values of the restoration. The degree of matching between the restoration and the tooth is
$74.5 \%$. A significant change was noted for all the specimens $(\mathrm{p}<0.001) . \Delta \mathrm{b}$ was in the range $(-15.7-$ $-0.6)$ mean $-7.84 \pm 3.7$. All the specimens tested showed negative $\Delta \mathrm{b}$ which indicates that shade is directed toward blue.

Color changes $(\Delta \mathrm{E})$ of the resin material were ranged from 1.2-17.7 $(9.89 \pm 4.86)$.

\section{DISCUSSION}

The search for natural-looking restorations led to the recent introduction of restorative systems containing several shades and different translucency levels, including enamel shade resin composites of high, medium, and low value ${ }^{7}$.

According to the manufacturer, Omnichroma does not contain pigment, and its color properties are based on structural color/s, a smart chromatic technology with the goal of controlling the optical properties of the resin composite. This approach has enabled the engineering of a resin composite that responds to light waves at a given frequency by perfectly reflecting a specific wavelength inside the tooth color space ${ }^{12}$. To express structural color, it is important that the filler of the composite consists of specific, single-sized spherical particles only. As is demonstrated, TOKUYAMA's research found that $260 \mathrm{~nm}$ spherical filler generates the a and $\mathrm{b}$ color parameter necessary to match natural teeth. Variations in the size and shape of the filler material can alter or impede the structural color phenomenon, and ultimately the composite's shadematching ability. Therefore, OMNICHROMA uses 260nm spherical filler (OMNICHROMA Filler) material exclusively ${ }^{11}$.

This study was attempted to determine the total color matching of restored teeth with resin composite. Previous studies have used of several dimensions without because the natural teeth have different morphology, irregularities in thickness of enamel and dentin as well as in the surface structure of natural teeth. It should be noted that the color of 
the teeth is mainly determined by the dentin and not the enamel, with the latter having a minor influence on the teeth color but its main influence on the color perception in terms of lightness ${ }^{13}$. In the current study to overcome this problem, a standardized class $\mathrm{V}$ cavity was prepared in the tooth specimens since the minimal enamel thickness in this area so the restoration will be affected by the dentin color.

As the true color parameters values are controversial, spectrophotometric reference is the most closely approximate the true color parameter values, the $\mathrm{L}^{*}, \mathrm{~A}^{*}$, and $\mathrm{B}^{*}$ values of the individual shade-measuring devices were compared with the spectrophotometric reference system. VITA Easyshade ${ }^{\circledR}$ device differed from the spectrophotometric reference only in the $\mathrm{L}^{*}$ coordinates but this difference is not significant ${ }^{14}$.

The CIE Lab color system used in this study is a recommended method for dental purposes. ${ }^{12}$ It characterizes the color based on human perception, and designates it according to 3 spatial coordinates, $\mathrm{L}, \mathrm{a}$, and $\mathrm{b}^{8}$.

According to the findings of the current study, it was observed that color change $\Delta \mathrm{E}$ between the initial composite resin disc and composite resin restoration was high. As in agreement with TOKUYAMA's research filler powder itself has no color, as demonstrated by its appearance on a white paper background. White light reflected by the white background is strong, which is why a structural color phenomenon is not visible on the white background ${ }^{11}$. The high color-matching of the universal shade composite might be attributed to its high translucency reflecting the shade of the surrounding walls, even with different shades and translucency ${ }^{5}$. This agreed with Paravina et al. who concluded that the BE increased with increasing the translucency ${ }^{4}$.

Regarding the parameters $\Delta \mathrm{L}, \Delta \mathrm{a}$, and $\Delta \mathrm{b}$, where the $\Delta \mathrm{L}$ parameter did not have a statistically significant difference, this result was in contradiction with that of , on the other hand Peter Mourouzis et al. suggested that the restoration had the same lightness value as intact tooth, as well as the difference was not noticeable to the human eye. The above is justified, because it is harder to perceive a color difference due to lightness between two specimens than a color difference due to hue ${ }^{15}$.

The review of the overall differences in color coordinates between the intact teeth and the restored teeth indicates that the difference in the lightness coordinate $\Delta \mathrm{L}$ had a positive value for all teeth, which means that there was a shift to a lighter shade. The difference in the red-green coordinate $(\Delta a)$ was statically significant with a negative value $(-a)$, which indicates shifting to the green scale. The difference in the blue-yellow coordinate $(\Delta b)$ was statically significant with negative value (-b), which indicates a shift to blue scale. This was contradictory to Dunn $\mathrm{k}$ et $\mathrm{al}^{16}$ compared $\mathrm{L}, \mathrm{a}, \mathrm{b}$ values the mean values of the restoration showed close numbers to the mean values of the surrounding enamel. Thus, supporting the color matching adaptation of OMNICHROMA ${ }^{11}$.

Other studies provided ratings of excellent or good for OMNICHROMA's color-matching ability with $60.8 \%$ ranking it as excellent ${ }^{16}$, this agreed with our study, which showed matching up to $75 \%$. This is may be due to differences in color detecting methods. In this study, which was in vitro study, we used digitalized method $\left(\right.$ Easyshade $^{\mathcal{O}}$ ) with numerical results. While in the other studies, which was in vivo study, they use pear eyes to detect the color matching level. On the contrary Rasha et $\mathrm{al}^{5}$ and Paravina et $\mathrm{al}^{4}$ who stated that the interand intra-observer agreement in their study were relatively high. This might be due to the $\mathrm{BE}$ of the extracted teeth differed from that of the composite, the difference in their nature; for example, natural teeth are multilayered and polychromatic ${ }^{4}$. 


\section{CONCLUSIONS}

\section{Under the limitation of the present study it could be concluded that}

1. The change in color of single shade resin composite between its initial color and as a restoration is remarkable.

2. The change in the brightness between the initial color and as a restoration indicates that the resin material became darker.

3. OMNICHROMA single shade resin composite matches the shade of enamel for class $\mathrm{V}$ restorations.

4. The blending effect of single shade structurally colored universal resin composite is promising.

\section{RECOMMENDATIONS}

It may be better to assess color-matching and blending effect of single shade resin composite in vivo as it is a better simulation of clinical condition.

\section{REFERENCES}

1. R Veena Kumari, Hema Nagaraj, Kishore Siddaraju, and Ramya Krishna Poluri. Evaluation of the Effect of Surface Polishing, Oral Beverages and Food Colorants on Color Stability and Surface Roughness of Nanocomposite Resins. J Int Oral Health. 2015 Jul; 7(7): 63-70.

2. Lee YK. Translucency of human teeth and dental restorative materials and its clinical relevance. J Biomed Opt. 2015 Apr;20(4):1-8.

3. Diogo de Azevedo Miranda, Yuri Lobo Valle Marçal, Fernanda Pícoli Proba, Thayane Kethlin Pimenta Moreira, Laura Nobre Ferraz, and Flávio Henrique Baggio Aguiar. Color correspondence of different brands and composite resin systems in relation to the Vita Classical scale through spectrophotometry. Dent Oral Craniofac Res.2018; 5(1): 1-4.

4. Rade D. Paravinaa, Stephen Westlandb, Mikio Kimuraa, John M. Powers, and Francisco H. Imaic. Color interaction of dental materials blending effect of layered composites. Dental materials.2006;22:903-8.

5. Rasha M. Abdelraouf and Nour A. Habib. Color matching and blending effect of Universal Shade Bulk-Fill resin composite in Resin Composite models and natural Teeth. BioMed Research International. 2016:1-8.

6. Andrew Joiner. Tooth Color: A Review of the Literature. 2004;32 Suppl 1:3-12.

7. M Schmeling, A Meyer-Filho MAC Andrada, and LN Baratieri. Chromatic Influence of Value Resin Composites. Operative Dentistry. 2010;35(1): 44-9.

8. Neslihan Tekçe, Safa Tuncer, Mustafa Demirci, Merve Efe Serim, Canan Baydemi. The Effect of Different Drinks on the Color Stability of Different Restorative Materials After One Month. Restor Dent Endod. 2015;40(4):255-61.

9. Kim BJ,Lee YK. Influence of the shade designation on the color difference between the same shade-designated resin composites by the brand. Dent Mater. 2009;25: 1148-54.

10. Natalie Pereira Sanchez, John M. Powers, and Rade D. Paravina. Instrumental and visual evaluation of the color adjustment potential of resin composites. J Esthet Restor Dent. 2019; 31:465-70.

11. TOKUYAMA, Omnichroma.

12. Paravina RD, Majkic G, Imai FH, Powers JM. Optimization of tooth color and shade guide design. J Prosthodont. 2007; 16:269-76.

13. Peter Mourouzis, Elisabeth A. Koulaouzidou, Georgios Palaghias, and Maria Helvatjoglu-Antoniades. Color match of resin composites to intact tooth structure. J Appl Biomater Funct Mater. 2015; 13(3): e259-e65.

14. Karl Martin Lehmann, Christopher Igiel, Irene Schmidtmann, Herbert Scheller. Four color-measuring devices compared with a spectrophotometric reference system. journal of dentistry. 2010;38s: e65 - e70.

15. Paravina RD, Kimura M, Powers JM. Color compatibility of resin composites of identical shade designation. Quintessence Int713-9:(9)37;2006.

16. Mohamed MA, Afutu R, Tran D, Dunn K, Ghanem J, and Perry R and Kugel G. Shade Matching Capacity of Omnichroma in Anterior Restorations. Journal of Dental Sciences. 2020 ;5(1):1-6. 